\title{
Even-odd filling-factor switching in one-dimensional lateral superlattices
}

\author{
M. Tornow, D. Weiss, ${ }^{*}$ A. Manolescu, ${ }^{\dagger}$ R. Menne, and K. v. Klitzing \\ Max-Planck-Institut für Festkörperforschung, Heisenbergstraße 1, D-70569 Stuttgart, Germany \\ G. Weimann \\ Fraunhofer Institut für angewandte Festkörperphysik, 79108 Freiburg, Germany
}

(Received 26 August 1996)

\begin{abstract}
The magnetoresistance of a two-dimensional electron system, subjected to a one-dimensional lateral potential modulation is studied for various potential amplitudes. The interplay between Landau level splitting and modulation broadening is shown to generate a transition from even to odd filling factors for the Shubnikov-de Haas resistance minima. The effect provides a tool to determine the potential amplitude. Calculations within a one-particle model agree with this evidence of the van Hove-like structure of the Landau bands. [S0163-1829(96)04748-0]
\end{abstract}

The magnetotransport properties of a two-dimensional electron system (2DES), subjected to a lateral onedimensional (1D) potential modulation, have been the subject of extensive work. Considerable insight has been achieved for ballistic transport in weak periodic potentials. In this case, the commensurability between the two length scales "classical cyclotron radius $R_{C}$ " and "modulation period $a$ " dominates the low magnetic field regime with pronounced $1 / B$ periodic oscillations ${ }^{1-3}$ which are followed by a positive magnetoresistance at higher $B{ }^{4}$ Increasing the modulation amplitude first leads to a damping of the resistance oscillations ${ }^{5,6}$ while in the limit of extremely strong modulation a pronounced negative magnetoresistance at low magnetic fields was found, accompanied by almost dissipationless transport in the quantum Hall regime. ${ }^{7}$

The one-dimensional potential modulation of a 2DES broadens the Landau levels into bands by lifting their degeneracy with respect to the center coordinate of the wave function. These Landau bands exhibit a peculiar density of states (DOS) with maxima at the band edges (van Hove singularities) and minima in the band centers. ${ }^{8}$ Measurements on 1D laterally modulated $\mathrm{GaAs} / \mathrm{Al}_{x} \mathrm{Ga}_{1-x}$ As heterostructures revealed already an unusual splitting of a single Shubnikov-de Haas $(\mathrm{SdH})$ maximum, which was ascribed to the double peak structure of the DOS in these systems. ${ }^{9}$ Recently observed complicated internal structure of a single Landau band was treated theoretically in an approach involving Coulomb interaction effects. ${ }^{10}$

In the present work we study the intermediate modulation amplitude regime in quantizing magnetic fields. When the cyclotron energy becomes comparable to the Landau bandwidth, i.e., when the bands start to overlap, a quenching of the oscillatory magnetoresistance is expected. The quenching of the $\mathrm{SdH}$ oscillations at low magnetic fields was used to estimate the modulation amplitude in previous work. ${ }^{5}$ Here however, we investigate the $\mathrm{SdH}$-like peaks at higher $B$ where we find a manifestation of the van Hove-like DOS. The transition from broadened Landau bands, separated by a gap, to overlapping ones generates a crossover from even to odd filling factors at the $\mathrm{SdH}$ minima positions, in good qualitative agreement with calculations of the resistivity. We assign the magnetic field position where the switching occurs to $V_{p p} \approx \hbar \omega_{C}$, where $V_{p p}$ denotes the peak-to-peak potential amplitude to be extracted from this effect. Note that this magnetic field position differs from the (lower) $B$ field where the SdH oscillations disappear. We also investigated the temperature dependence of the $\mathrm{SdH}$ oscillations and extracted the activation energy in the Landau gap at filling factor $\nu \approx 2$ for different modulation strength. The corresponding amplitudes there exceed by more than a factor of two our low-field estimates $V_{p p}$ what we ascribe to the reduced screening due to the small DOS at the Fermi level.

We start from a modulation doped GaAs/ $\mathrm{Al}_{x} \mathrm{Ga}_{1-x} \mathrm{As}$ heterostructure with carrier density $n_{s}=3.3 \times 10^{15} \mathrm{~m}^{-2}$ and mobility $\mu=31 \mathrm{~m}^{2} / \mathrm{Vs}$ at $T=4.2 \mathrm{~K}$ measured in the dark. On top of a conventionally prepared Hall bar geometry, we patterned a 1D resist grating of period $a=500 \mathrm{~nm}$ by using electron beam lithography. Subsequent wet chemical etching $\left(\mathrm{H}_{2} \mathrm{O}_{2}\right.$ buffered to $\mathrm{pH} 7$ with $\mathrm{NH}_{4} \mathrm{OH}$ at $\left.5{ }^{\circ} \mathrm{C}\right)$ transferred the grating into the device by removing selectively the thin GaAs cap layer only (see left inset of Fig. 3). As a result of the different band bending at the surface, the carrier density beneath the etched regions is reduced, leading to a lateral modulation potential for the conduction electrons. By illuminating the device with a red light-emitting diode (LED) at different intensities, either by a saturating pulse or continuously, ${ }^{11}$ the modulation amplitude could be adjusted over a wide range at low temperatures. Here we use the persistent photoconductivity effect at low temperatures and the charge redistribution within the surface depletion layer by a (steady) photoexcitation of electrons and holes. ${ }^{7}$ Magnetotransport experiments have been carried out at temperatures between $1.4 \mathrm{~K}$ and $20 \mathrm{~K}$ in magnetic fields up to $12 \mathrm{~T}$. We applied an ac (10-100 nA) perpendicular to the grating and measured the voltage drop by lock-in techniques to obtain the resistivity $\rho_{x x}$.

Figure 1 displays magnetoresistance data from a single sample at four different modulation strengths, taken at $1.4 \mathrm{~K}$. At low magnetic fields $(<0.5 \mathrm{~T})$, only a remainder of the commensurability oscillations is resolved due to the strong modulation. ${ }^{5}$ However, the electron motion is still ballistic over at least one period with elastic mean free paths of 0.7 

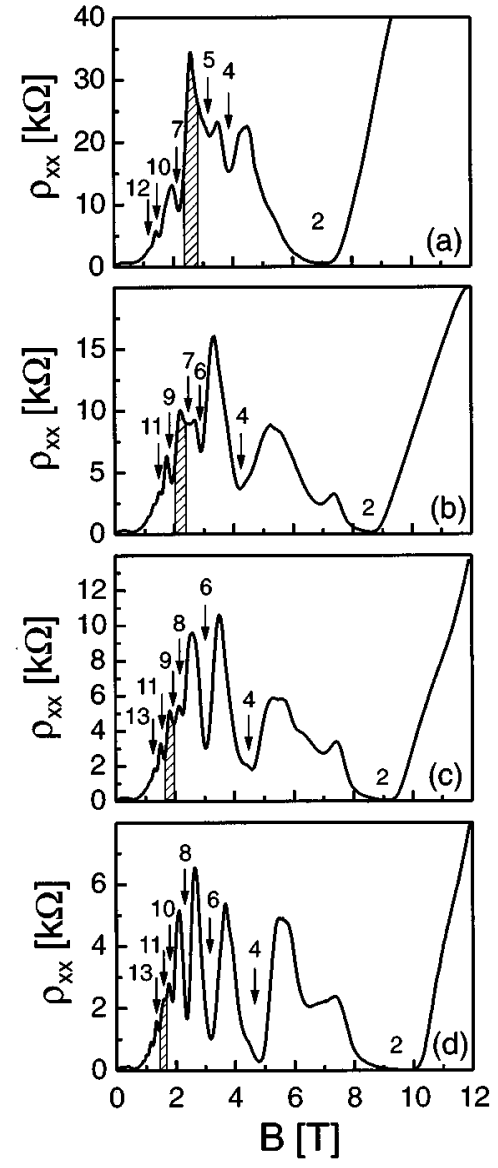

FIG. 1. Magnetoresistance at $1.4 \mathrm{~K}$ of 1D laterally modulated 2DES for four different modulation amplitudes, adjusted by a differently intense illumination by a LED: (a) $V_{p p}=4.5 \mathrm{meV}$, (b) $V_{p p}=3.8 \mathrm{meV}$, (c) $V_{p p}=3.1 \mathrm{meV}$, (d) $V_{p p}=2.7 \mathrm{meV}$. Numbers denote Landau level filling factors corresponding to $\mathrm{SdH}$ minima, marked by arrows. The vertical dashed bars mark the lowest even filling factor $\mathrm{SdH}$ maxima used to determine the modulation strength.

$\mu \mathrm{m}, 2.0 \mu \mathrm{m}, 2.4 \mu \mathrm{m}$, and $2.9 \mu \mathrm{m}$ as can be extracted from the zero-field resistivities of the data displayed in Figs. 1(a)(d), respectively. This justifies rather a picture of electron transport in energy bands than transport across a series of independent stripes with different Ohmic resistivities. At slightly higher fields the resistance increases-apart from the oscillatory behavior-quadratically. ${ }^{12}$

For all different potential amplitudes, quantum oscillations are observable above $1 \mathrm{~T}$ in Fig. 1. Their shape differs considerably from the one of conventional $\mathrm{SdH}$ oscillations in an unmodulated system. To obtain information about the origin of the oscillations we plotted the magnetic field position of the $\rho_{x x}$ minima in the usual "Landau plot" vs $1 / B$, counting the number $\nu=n_{\mathrm{s}} h / e B$ of occupied (spin split) Landau levels. The results are displayed in Fig. 2. At high magnetic fields even filling factors, corresponding to the Landau gaps in the density of states, fit a straight line, resembling the carrier density. Proceeding to higher Landau level indices, one finds the remarkable result, that the filling factors of the minima suddenly switch to odd. These minima at odd filling factors are not related to spin splitting of the Landau levels which cannot be resolved at the low magnetic

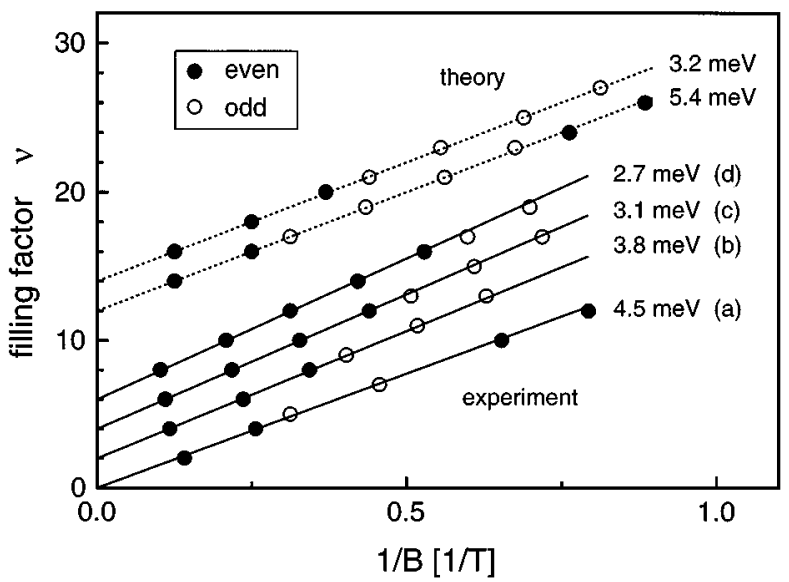

FIG. 2. Landau level filling factor versus SdH minimum position in $1 / B$ for the four investigated modulation strengths (full lines) and from the calculated examples (dashed lines). Labels (a)-(d) correspond to Fig. 1. Full and empty circles denote even and odd filling factors, respectively. Curves are offset for clarity.

fields where the switching occurs. This has been confirmed in an experiment, where the 2DES plane was tilted against the magnetic field direction. We ascribe the minima at odd filling factors to the reduced DOS at the centers of modulation broadened Landau bands which follow the imposed potential [see Figs. 4(b)-(g)]. The resistance maxima hence appear at even filling factors and correspond to overlapping van Hove singularities at the edges of adjacent Landau bands. They are especially pronounced at the $B$ positions of just disappearing Landau gaps. In Fig. 1 the first of these maxima-coming from high magnetic fields-is marked with a hatched bar. At this magnetic field position we assume that $\hbar \omega_{C} \approx V_{p p}$ holds. Clearly, for reduced modulation potential this condition shifts to lower $B$ [from Fig. 1(a) to Fig. $1(\mathrm{~d})$ ]. We use this coincidence to evaluate the present modulation amplitudes to be $V_{p p}=4.5,3.8,3.1$, and $2.7 \mathrm{meV}$, respectively.

For all four modulation strengths the temperature dependence of the magnetoresistance has been measured between $1.4 \mathrm{~K}$ and $20 \mathrm{~K}$. Figure 3 displays as an example the result for $V_{p p}=3.8 \mathrm{meV}$. As expected, the classical background at low $B$ is stable with the temperature. At high $B$, the resistance minima at even filling factors show a strong resistance decrease with decreasing temperature. In the simplest picture we expect a thermally activated resistivity ${ }^{13}$ with an activation energy $E_{A} \approx 0.5\left(\hbar \omega_{C}-V_{p p}\right)$. The right inset of Fig. 3 shows $E_{A}$ as a function of $V_{p p}$, extracted from the temperature dependence of the $\nu \approx 2$ minima. Here, the $E_{A}$ values have been evaluated at those magnetic field positions which yield the maximal slope of the Arrhenius plots. These magnetic field positions (see dashed line in Fig. 3) do not necessarily coincide with the "exact" minima positions at $T=1.4 \mathrm{~K}$. With increasing $V_{p p}$ the activation energy $E_{A}$ decreases as expected from the picture described above. For higher modulation amplitudes however, the gap seems to be smaller than expected from estimating $V_{p p}$ at lower $B$. We anticipate that this deviation might be due to screening of the potential by the $2 \mathrm{D}$ electron gas. At lower $B$ where the bands overlap, the DOS is finite and the estimated potential $V_{p p}$ is therefore a well screened one. In contrast, in the Landau gap 


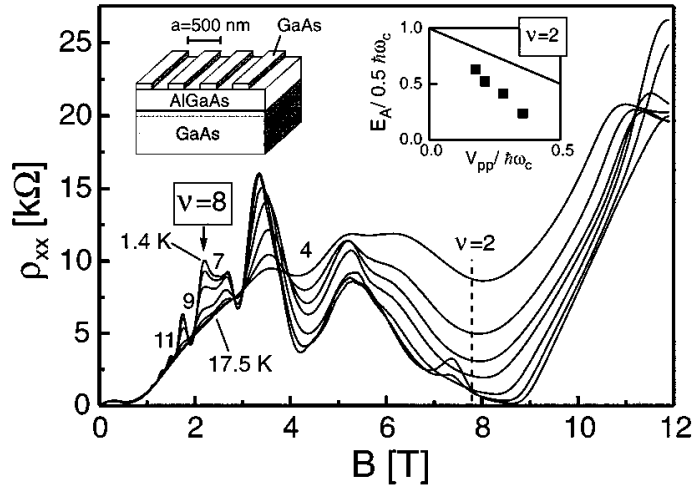

FIG. 3. Temperature dependence of the magnetoresistance for the sample with $V_{p p}=3.8 \mathrm{meV}$. Temperatures are: $1.4 \mathrm{~K}, 2.0 \mathrm{~K}$, 4.2 K, 7.5 K, $10.5 \mathrm{~K}, 13.5 \mathrm{~K}$ and $17.5 \mathrm{~K}$. The position of filling factor $\nu \approx 2$ used to evaluate the activation energy $E_{A}$ is marked with a dashed line. Left inset: Schematical drawing of the GaAs/ $\mathrm{Al}_{x} \mathrm{Ga}_{1-x} \mathrm{As}$ heterostructure after the selective removal of caplayer GaAs stripes. Right inset : $E_{A}$ as a function of $V_{p p}$ at $\nu \approx 2$, normalized with respect to the (half) cyclotron energy. The line sketches the dependence expected from a $B$-independent modulation amplitude (see text).

around $\nu \approx 2$, the DOS becomes small which leads to a poor screening of the external potential. Hence, the resulting bandwidth at $\nu \approx 2$ will be larger than $V_{p p}$, reflected in a reduced $E_{A}$. We note that a realistic quantitative analysis of the temperature dependence remains beyond the scope of this work, ${ }^{14}$ since the screening itself is temperature dependent. $^{15,16}$

Approaching from the high $B$ side (Fig. 3, main viewgraph) the transition from separated to overlapping bands, the (activated) temperature dependence gets less and less pronounced, resembling lower and lower $E_{A}$. Finally, neighboring van Hove singularities touch and the first resistance maximum at even filling factor ( $\nu=8$ in Fig. 3) is resolved. The resistance minima in the overlap region show a temperature dependence quite contrary to those of the Landau gaps. They are either almost stable with the temperature $(\nu=9$, 11) or even increase in their absolute value $(\nu=7)$, when decreasing the temperature. We take this as an additional confirmation that the density of states is finite in this region rather than showing a real gap. This behavior allows us to distinguish from a second subband occupation (see, e.g., Ref. 17), where a change from even to odd filling factors occurs as well.

To check the picture given above for the observed crossover from even to odd filling factors, we calculated the resistivity $\rho_{x x}$ for a modulation potential of the form $V(x)=0.5 V_{p p} \cos (2 \pi x / a)$, assuming that the higher harmonics of the electrostatic potential at the heterostructure surface are exponentially attenuated. ${ }^{15}$ In Fig. 3 the second Landau band (between filling factor 2 and 4) starts to reveal internal structure at the lowest temperatures. In this $B$ regime we expect a complicated interplay between van Hove singularity formation in the DOS and enhanced spin splitting, not considered here (see, e.g., Refs. 10 and 16). At intermediate and weak fields, addressed in the present work, the picture of noninteracting electrons seems to be sufficient for a qualitative explanation of the effects observed. Including the scat-
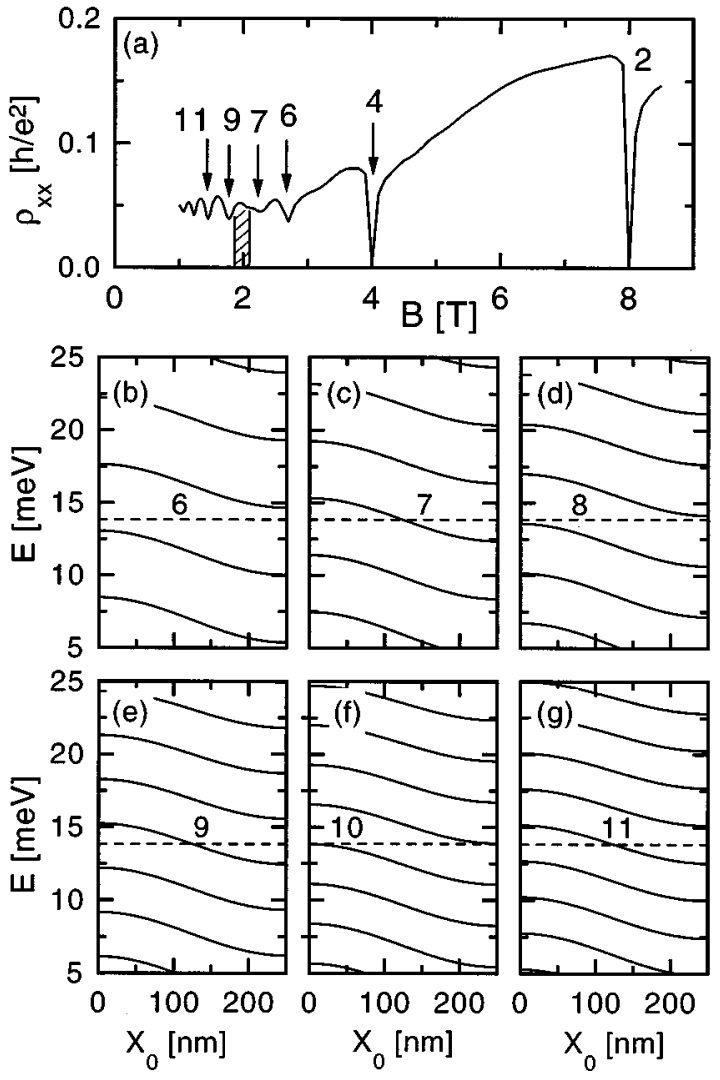

FIG. 4. (a) Calculated magnetoresistance for $V_{p p}=3.2 \mathrm{meV}$ with $n_{s}=3.86 \times 10^{15} \mathrm{~m}^{-2}(\nu B=16 \mathrm{~T}), a=500 \mathrm{~nm}, \gamma=0.6$, and $T=2 \mathrm{~K}$. The energy spectra (b) $-(\mathrm{g})$ are plotted as a function of the center coordinate $X_{0}$ in half of a period for decreasing magnetic field values. Thus, they illustrate the mechanism of even-odd switching of the $\rho_{x x}$ minima. The numbers 6 to 11 stand for the filling factor at the Fermi energy which is shown as a dashed line.

tering broadening $\Gamma$ in a phenomenological self-consistent Born approximation $[\Gamma=\gamma \sqrt{B(\text { Tesla })}(\mathrm{meV})]$, we calculated the resistivity $\rho_{x x}$ using a Kubo formalism. ${ }^{8}$

In Fig. 4(a) we show the calculated resistivity $\rho_{x x}$ for $V_{p p}=3.2 \mathrm{meV}$. Except at high magnetic fields (localization effects, etc., are not included), the calculated resistivity is in good qualitative agreement with experiment, as will be pointed out below. As in experiment, the $B$ values at which even-odd switching occurs shift to higher magnetic field (lower $\nu$ 's) with increasing modulation strength (see upper traces in Fig. 2). The resistivity oscillations are determined by the oscillations of the DOS at the Fermi level. The latter can be deduced from the energy spectra, displayed in Figs. 4(b)-(g). In Fig. 4(b) three Landau levels are occupied (filling factor 6) and $E_{F}$ is located in the gap, giving rise to a $\rho_{x x}$ minimum in the resistivity [Fig. 4(a)]. The minimum labeled 7 already corresponds to a position of $E_{F}$ at the Landau band center, where the DOS is reduced with respect to the band edges [Fig. 4(c).$^{18}$ In Fig. 4(d) finally $V_{p p} \approx \hbar \omega_{C}$ holds and $E_{F}$ is "attached" to the van Hove singularities of adjacent bands, giving rise to a $\rho_{x x}$ maximum. It is this situation that we use in experiment to determine $V_{p p}$. By further reducing $B$ we stay within the regime of overlapping Landau bands and observe as in experiment further minima at odd (band centers) and maxima at even filling factors (overlapping band edges). 
Note the particular situation for the strongest modulation in experiment [Fig. 1(a)], where after the switching to odd filling factors with decreasing $B$ a second change back to even numbers is observed. To explain such behavior, the overlapping of three Landau bands has to be taken into ac- count. As an example, such a situation was calculated for $V_{p p}=5.4 \mathrm{meV}$ and is included into Fig. 2 .

We thank C. Albrecht, R. R. Gerhardts, and P. D. Ye for useful discussions and M. Riek and U. Waizmann for technical support.
*Present address: Experimentelle und Angewandte Physik, Universität Regensburg, D-93 040 Regensburg, Germany.

†Present address: Institutul de Fizica şi Tehnologia Materialelor, C.P. MG-7 Bucureşti-Măgurele, Romania.

${ }^{1}$ D. Weiss, K. v. Klitzing, K. Ploog, and G. Weimann, Europhys. Lett. 8, 178 (1987); R. R. Gerhardts, D. Weiss, and K. v. Klitzing, Phys. Rev. Lett. 62, 1173 (1989).

${ }^{2}$ R. W. Winkler, J. P. Kotthaus, and K. Ploog, Phys. Rev. Lett. 62, 1177 (1989).

${ }^{3}$ C. W. J. Beenakker, Phys. Rev. Lett. 62, 2020 (1989).

${ }^{4}$ A. K. Geim, R. Taboryski, A. Kristensen, S. V. Dubonos, and P. E. Lindelof, Phys. Rev. B 46, 4324 (1992).

${ }^{5}$ P. H. Beton, E. S. Alves, P. C. Main, L. Eaves, M. W. Dellow, M. Henini, O. H. Hughes, S. P. Beaumont, and C. D. W. Wilkinson, Phys. Rev. B 42, 9229 (1990).

${ }^{6}$ C. Nguyen, B. Brar, V. Jayaraman, A. Lorke, and H. Kroemer, Appl. Phys. Lett. 63, 2251 (1993).

${ }^{7}$ G. Müller, P. Streda, D. Weiss, K. v. Klitzing, and G. Weimann, Phys. Rev. B 50, 8938 (1994); G. Müller, D. Weiss, K. v. Klitzing, P. Streda, and G. Weimann, ibid. 51, 10236 (1995).

${ }^{8}$ C. Zhang and R. R. Gerhardts, Phys. Rev. B 41, 12850 (1990).

${ }^{9}$ D. Weiss, K. v. Klitzing, K. Ploog, and G. Weimann, Surf. Sci.
229, 88 (1990).

${ }^{10}$ A. Manolescu, R. R. Gerhardts, M. Tornow, D. Weiss, K. v. Klitzing, and G. Weimann, Surf. Sci. 361/362, 513 (1996).

${ }^{11}$ J. G. Michels, R. J. Nicholas, G. M. Summers, D. M. Symons, C. T. Foxon, and J. J. Harris, Phys. Rev. B 52, 2688 (1995).

${ }^{12}$ The strength of the quadratic rise was used previously to estimate the amplitude of the potential modulation, see Ref. 4. This evaluation assumes a weak potential $\left(V_{p p} \ll E_{F}\right.$, with $E_{F}$ the Fermi energy) and cannot be applied here.

${ }^{13}$ E. Stahl, D. Weiss, G. Weimann, K. v. Klitzing, and K. Ploog, J. Phys. C 18, L783 (1985).

${ }^{14}$ Experimentally, the evaluation of $E_{A}$ is complicated by the fact that our samples show a small (temperature dependent) parallel conductivity.

${ }^{15}$ U. Wulf, V. Gudmundsson, and R. R. Gerhardts, Phys. Rev. B 38, 4218 (1988).

${ }^{16}$ A. Manolescu and R. R. Gerhardts, Phys. Rev. B 51, 1703 (1995).

${ }^{17}$ H. van Houten, J. G. Williamson, M. E. I. Broekaart, C. T. Foxon, and J. J. Harris, Phys. Rev. B 37, 2756 (1988).

${ }^{18}$ The band-center DOS at lower filling factors $(\nu=5,3)$ does not show relative minima due to the increased scattering broadening at higher $B$. 